\title{
Dark energy in motion*
}

\author{
Antonio L. Maroto \\ Departamento de Física Teórica \\ Universidad Complutense de Madrid \\ 28040 Madrid, Spain
}

\begin{abstract}
Recent large-scale peculiar velocity surveys suggest that large matter volumes could be moving with appreciable velocity with respect to the CMB rest frame. If confirmed, such results could conflict with the Cosmological Principle according to which the matter and CMB rest frames should converge on very large scales. In this work we explore the possibility that such large scale bulk flows are due, not to the motion of matter with respect to the $\mathrm{CMB}$, but to the flow of dark energy with respect to matter. Indeed, when dark energy is moving, the usual definition of the CMB rest frame as that in which the $\mathrm{CMB}$ dipole vanishes is not appropriate. We find instead that the dipole vanishes for observers at rest with respect to the cosmic center of mass, i.e. in motion with respect to the background radiation.
\end{abstract}

* Essay selected for "Honorable Mention" in the 2006 Awards for Essays on Gravitation (Gravity Research Foundation) 
In Standard Cosmology, the universe is assumed to be homogeneous and isotropic on very large scales. In smaller volumes, the presence of density inhomogeneities generates deviations in the motion of galaxies with respect to the pure Hubble flow. The amplitude of such peculiar velocities is determined by the amplitude of the density perturbations. However, as we take larger and larger averaging volumes, we expect, according to the Cosmological Principle, the density contrasts to decline and consequently the velocity fields to converge towards the pure Hubble flow. In other words, at large scales, the matter rest frame should converge to the frame in which the expansion is isotropic, i.e. to the frame in which the CMB dipole anisotropy vanishes [1].

However as shown in Fig. 1, this theoretical framework is not conclusively confirmed by observations. Indeed, in recent years several peculiar velocity surveys [2] have tried to determine the volume size at which the streaming motion of matter with respect to the CMB vanishes. In the figure the results of different observations are compared with the $r m s$ expected bulk velocity $V_{b}$ for standard $\Lambda$ CDM model in a sphere of radius $R$. The results seem to agree with the theoretical expectations only at scales $R \lesssim 60 h^{-1}$ Mpc. At larger scales, $R \gtrsim 100 h^{-1} \mathrm{Mpc}$, different data sets lead to different bulk velocities both in amplitude and direction. Moreover, there are indeed measurements in which large matter volumes are moving at speeds $\gtrsim 600 \mathrm{~km} \mathrm{~s}^{-1}$ with respect to the CMB frame, several standard deviations away from the theoretical predictions. These results have been argued to be affected by systematic errors in distance indicators, but if confirmed by future surveys, a revision of some of the underlying ideas in Standard Cosmology would be required in order to understand the origin of such large flows.

On the other hand, the main contribution to the CMB dipole anisotropy 


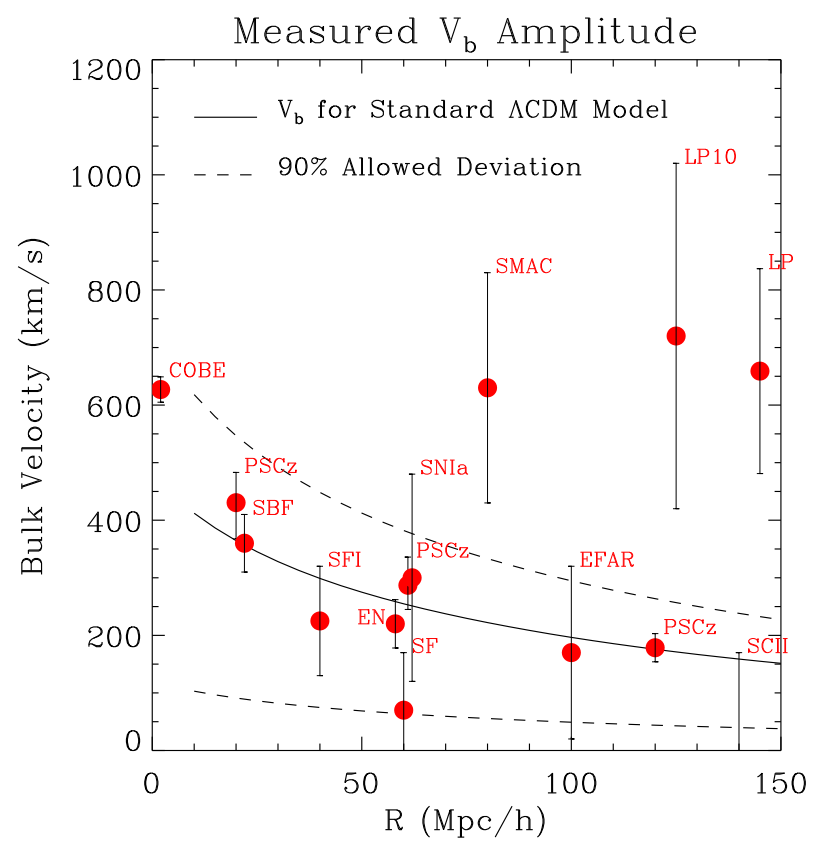

Figure 1: Bulk flow measurements from different peculiar velocity surveys [2]. The solid line corresponds to the expected rms velocity in the standard $\Lambda$ CDM cosmology, together with the $90 \%$ deviation in dashed lines. Figure from S. Zaroubi [3]

is usually attributed to a Doppler effect induced by the motion of the observer with respect to the last scattering surface [1]. For that reason, bulk flow velocities are understood as the average velocity of a given matter volume with respect to an observer who measures a vanishing CMB dipole. Accordingly, a different origin of the CMB dipole would have important consequences for the determination of cosmic flows.

So far we have only been concerned with the possibility that matter and radiation could have different rest frames even when averaged over large volumes. However matter and radiation are not the dominant components of the universe today. We know from high-redshift supernovae and other cosmological observations, that the universe is dominated at present by some 
sort of dark energy with negative pressure and whose nature is still a mistery. Several models have been proposed for dark energy such as a pure cosmological constant, quintessence or k-essence scalar fields. In all those cases, dark energy is completely decoupled from matter and radiation, its only effects being of gravitational nature.

The existence of a common rest frame is expected for strongly coupled fluids, as is indeed the case for baryonic matter and radiation before recombination. However, this might not be true at the present epoch when matter, radiation, and presumably dark energy are almost completely decoupled. In such a case, it makes sense to explore the possibility that the different components have different rest frames. In the following we will show that in such a case, the usual interpretation of the CMB dipole is not appropriate and that even an observer at rest with respect to the CMB could observe a non-vanishing dipole, provided dark energy is moving with respect to matter. This opens the interesting possibility of having non-vanishing bulk flow velocities at large scales even if matter and radiation share a common rest frame.

Let us therefore consider a cosmological scenario with three perfect fluids: radiation, matter and dark energy, whose equations of state read $p_{\alpha}=w_{\alpha} \rho_{\alpha}$ with $\alpha=R, M, \Lambda$. For the sake of generality, we will allow the dark energy equation of state to have a smooth dependence on redshift $w_{\Lambda}(z)$. The energy-momentum tensor of each fluid will take the form:

$$
\left(T^{\mu}{ }_{\nu}\right)_{\alpha}=\left(\rho_{\alpha}+p_{\alpha}\right) u_{\alpha}^{\mu} u_{\nu \alpha}-p_{\alpha} \delta_{\nu}^{\mu}
$$

Since we are only interested in the effects of fluids motion on the CMB dipole, it is sufficient to take into account the evolution of the largest-scale velocity perturbations, i.e. we will just consider the zero-mode equations. The pres- 
ence of inhomogeneities will contribute to higher multipoles. Therefore, for this particular problem we can write:

$$
\begin{aligned}
\rho_{\alpha} & =\rho_{\alpha}(\eta), \\
p_{\alpha} & =p_{\alpha}(\eta), \\
u_{\alpha}^{\mu} & =\frac{1}{a}\left(1, v_{\alpha}^{i}(\eta)\right)
\end{aligned}
$$

We will assume that $\vec{v}_{\alpha}^{2} \ll 1$ and we will work at first order in perturbation theory. In the particular case we are considering, the form of the spacetime metric will be given by the following vector-perturbed spatially-flat Friedmann-Robertson-Walker metric:

$$
d s^{2}=a^{2}(\eta)\left(d \eta^{2}+2 S_{i}(\eta) d \eta d x^{i}-\delta_{i j} d x^{i} d x^{j}\right)
$$

Accordingly, the total energy-momentum tensor reads:

$$
\begin{aligned}
T_{0}^{0} & =\sum_{\alpha} \rho_{\alpha} \\
T_{i}^{0} & =\sum_{\alpha}\left(\rho_{\alpha}+p_{\alpha}\right)\left(S_{i}-v_{i \alpha}\right) \\
T^{i}{ }_{0} & =\sum_{\alpha}\left(\rho_{\alpha}+p_{\alpha}\right) v_{\alpha}^{i} \\
T_{j}^{i} & =-\sum_{\alpha} p_{\alpha} \delta^{i}{ }_{j}
\end{aligned}
$$

Notice that we are considering only the epoch after matter-radiation decoupling, assuming that dark energy is also decoupled and for that reason we will ignore possible energy and momentum transfer effects.

We now calculate the linearized Einstein equations using (3) and (4). They yield just the condition:

$$
S^{i}=\frac{\sum_{\alpha}\left(\rho_{\alpha}+p_{\alpha}\right) v_{\alpha}^{i}}{\sum_{\alpha}\left(\rho_{\alpha}+p_{\alpha}\right)}
$$


In General Relativity the combination $\left(\rho_{\alpha}+p_{\alpha}\right)$ appearing in (5) plays the role of inertial mass density of the corresponding fluid, and accordingly $\vec{S}$ can be understood as the cosmic center of mass velocity. Notice that a pure cosmological constant has no inertial mass density.

On the other hand, the energy conservation equations are trivially satisfied, whereas from momentum conservation we see that the velocity of each fluid relative to the center of mass frame scales as: $\left|\vec{S}-\vec{v}_{\alpha}\right| \propto a^{3 w_{\alpha}-1}$. Notice that for dark energy the scaling properties will depend on the particular model under consideration [4].

Once we know the form of the perturbed metric, we can calculate the effect of fluids motion on photons propagating from the last scattering surface using standard tools [5]. The energy of a photon coming from direction $n^{\mu}=\left(1, n^{i}\right)$ with $\vec{n}^{2}=1$ as seen by an observer moving with velocity $u^{\mu}=$ $a^{-1}\left(1, v^{i}\right)$ is given by $\mathcal{E}=g_{\mu \nu} u^{\mu} P^{\nu}$, i.e. to first order in the perturbation:

$$
\mathcal{E} \simeq \frac{E}{a}\left(1+\frac{d \delta x^{0}}{d \eta}+\vec{n} \cdot(\vec{S}-\vec{v})\right)
$$

where $E$ parametrizes the photon energy and the perturbed trajectory of the photon reads $x^{\mu}(\eta)=x_{0}^{\mu}(\eta)+\delta x^{\mu}$, with $x_{0}^{\mu}=n^{\mu} \eta$.

In order to obtain $d \delta x^{0} / d \eta$, we solve the geodesics equations to first order in the perturbations, and for the 0-component we get $d^{2} \delta x^{0} / d \eta^{2}=0$. By defining $\hat{\mathcal{E}}=a \mathcal{E}$, the temperature fluctuation reads:

$$
\begin{aligned}
\left.\frac{\delta T}{T}\right|_{\text {dipole }} & =\left.\frac{\hat{\mathcal{E}}_{0}-\hat{\mathcal{E}}_{d e c}}{\hat{\mathcal{E}}_{d e c}} \simeq \frac{d \delta x^{0}}{d \eta}\right|_{d e c} ^{0}+\left.\vec{n} \cdot(\vec{S}-\vec{v})\right|_{d e c} ^{0} \\
& \left.\simeq \vec{n} \cdot(\vec{S}-\vec{v})\right|_{\text {dec }} ^{0}
\end{aligned}
$$

where the indices 0, dec denote the present and decoupling times respectively.

At decoupling, the universe is matter dominated and we can neglect the contribution to $\vec{S}$ from dark energy. We also assume that the velocity of 
matter is the same as that of radiation, and accordingly we have $\vec{S}_{d e c} \simeq \vec{v}_{M}^{d e c} \simeq$ $\vec{v}_{R}^{d e c}$. Here we are assuming for simplicity that baryonic and dark matter share a common rest frame. On the other hand, neglecting contributions to the CMB dipole of intrinsic density fluctuations in the last scattering surface, we can take the emitter velocity to be $\vec{v}_{d e c} \simeq \vec{v}_{M}^{d e c}$. So that we find:

$$
\begin{aligned}
\left.\frac{\delta T}{T}\right|_{\text {dipole }} & \simeq \vec{n} \cdot\left(\vec{S}_{0}-\vec{v}_{0}\right) \\
& \simeq \vec{n} \cdot \frac{\Omega_{M}\left(\vec{v}_{M}^{0}-\vec{v}_{0}\right)+\left(1+w_{\Lambda}^{0}\right) \Omega_{\Lambda}\left(\vec{v}_{\Lambda}^{0}-\vec{v}_{0}\right)}{1+w_{\Lambda}^{0} \Omega_{\Lambda}}
\end{aligned}
$$

where $w_{\Lambda}^{0}=w_{\Lambda}(0)$ is the present value of the dark energy equation of state and we have taken into account that today the contribution of radiation to the energy density is negligible.

According to this result, the CMB dipole is due to the relative velocity of the observer with respect to the present cosmic center of mass. When all the components share a common rest frame then the previous result reduces to the usual expression for the dipole: $\delta T /\left.T\right|_{\text {dipole }} \simeq \vec{n} \cdot\left(\vec{v}_{R}^{0}-\vec{v}_{0}\right)$. However in general it is possible that an observer at rest with radiation $\vec{v}_{0}=\vec{v}_{R}^{0} \neq \vec{v}_{M}^{0} \neq$ $\vec{v}_{\Lambda}^{0}$ can measure an nonvanishing dipole according to (8).

In the absence of dark energy or in the case in which it is in the form of a pure cosmological constant $\left(w_{\Lambda}=-1\right)$, dark energy would not contribute to the center of mass motion. Moreover, today the radiation contribution is negligible and accordingly the center of mass rest frame would coincide with the matter rest frame. This implies that the relative motion of matter and radiation today could not explain the existence of bulk flows on the largest scales, since the frame in which the dipole vanishes would coincide with the matter rest frame. Conversely, the existence of non-vanishing bulk flows would require the presence of moving dark energy with $w_{\Lambda}^{0} \neq-1$. 
Indeed, if moving dark energy is responsible for the existence of cosmic bulk flows on very large scales, then the amplitude and direction of such flows would provide a direct measurement of the relative velocity of matter and dark energy. As commented above, the bulk flow $\vec{V}_{b}$ can be understood as the average velocity of a given matter volume with respect to an observer who measures a vanishing CMB dipole, i.e. $\vec{V}_{b}=\vec{v}_{M}^{0}-\vec{v}_{0}$. Such an observer has a velocity which is given, according to (8), by:

$$
\vec{v}_{0} \simeq \vec{v}_{M}^{0}+\frac{\left(1+w_{\Lambda}^{0}\right) \Omega_{\Lambda}}{1+w_{\Lambda}^{0} \Omega_{\Lambda}}\left(\vec{v}_{\Lambda}^{0}-\vec{v}_{M}^{0}\right)
$$

so that

$$
\vec{V}_{b} \simeq \frac{\left(1+w_{\Lambda}^{0}\right) \Omega_{\Lambda}}{1+w_{\Lambda}^{0} \Omega_{\Lambda}}\left(\vec{v}_{M}^{0}-\vec{v}_{\Lambda}^{0}\right)
$$

Notice that, according to these results, even if matter is at rest with respect to the CMB radiation, $\vec{v}_{M}^{0}=\vec{v}_{R}^{0}$, it would be possible to have a non-vanishing flow $\vec{V}_{b} \neq 0$, provided dark energy is moving with respect to matter.

As commented above, matter and radiation were strongly coupled in the past and for that reason it is difficult to understand the presence of relative motions on very large scales. However, if the nature of dark energy is really unrelated to the rest of components of the universe, then the corresponding primordial dark energy bulk velocity should be considered as a free cosmological parameter. That such a primordial dark energy flow could have survived until present giving rise to the effects studied in this work is a fascinating possibility [4]. 
Acknowledgements: This work has been partially supported by the DGICYT (Spain) under the project numbers FPA 2004-02602 and FPA 200502327.

\section{References}

[1] P.J.E. Peebles and D.T. Wilkinson, Phys. Rev. 174, 2168 (1968); P.J.E. Peebles, Principles of Physical Cosmology, Princeton University Press (1993); A. Kogut et al., Astrophys.J. 419, 1 (1993)

[2] A.G. Riess et al., Astrophys. J. 488, L1 (1997); D.A. Dale et al. Astrophys.J. 510, L11 (1999); M. Colles et al., MNRAS, 321, 277 (2001); T.R. Lauer and M. Postman, Astrophys.J. 425, 418 (1994); M.J. Hudson et al. Astrophys.J. 512, L79 (1999); J.A. Willick, Astrophys.J. 522, 647 (1999); M.J. Hudson et al., Mon. Not. Roy. Astron. Soc. 352, 61 (2004);

M.J. Hudson, astro-ph/0311072

[3] S. Zaroubi, astro-ph/0206052. Contribution to XIII Recontres de Blois "Frontiers of the Universe", 17-23 June 2001

[4] A. L. Maroto, astro-ph/0512464. To be published in JCAP

[5] M. Giovannini, Int. J. Mod. Phys. D14, 363, (2005) 